\title{
Research Methodology for Enterprise Interoperability Architecture Approach
}

\author{
Wided Guédria $^{1}$, Khaled Gaaloul ${ }^{1}$, Henderik A. Proper ${ }^{1,2}$, \\ and Yannick Naudet ${ }^{1}$ \\ 1 Centre de Recherche Public Henri Tudor, Luxembourg \\ 2 Radboud University Nijmegen, Nijmegen, The Netherlands \\ \{wided.guedria, khaled.gaaloul, erik.proper, yannick. naudet\}@tudor.lu
}

\begin{abstract}
As technology becomes more far-reaching and interconnected, the need of interoperability is becoming increasingly important. The Ontology of Enterprise Interoperability (OoEI) was defined as a scientific reference model regarding interoperability leading to a common understanding involving this topic. The OoEI was proposed in the general context of Enterprise Interoperability with high level concepts using the system theory. This needs to be enriched with concepts from Enterprise domain. The discipline of Enterprise Architecture (EA) advocates the use of models to support business services on enterprises. Among them, this work focuses on ArchiMate. In order to provide business services support, ArchiMate, should be amenable to analyze of various properties, as e.g. the interoperability requirements. This paper proposes a set of concepts covering the EA and interoperability domains. Through literature review and framework research, we identify key aspects of interoperability and EA and their associations, resulting in a reference conceptual model for integrated Enterprise Architecture Interoperability. The proposed model is defined based on the Design Science Research methodology. A case study illustration will be used for the evaluation as part of the research approach.
\end{abstract}

Keywords: Research methodology, Enterprise Interoperability, Enterprise architecture.

\section{Introduction}

The obligation to become more competitive and effective in providing better products and services requires enterprises to interoperate and evolve into network. The availability of the Internet and information technologies has encouraged new business strategies that take advantage of enterprises ability to create networks or to network with other enterprises [1]. One of the challenges faced by a network of enterprises is the development of interoperability between its members, which is highly correlated to the ability to use networked architectures to collaborate efficiently [2,3].

Enterprise Architecture (EA) is generally considered to provide a good steering instrument to analyze the current state of the enterprise (As-is), identify and 
describe alternative future states (To-be), guard the cohesion and alignment between the different aspects of an enterprise such as business processes and their ICT (Information and Communications Technology) support [4. The analysis of the As-is situation is very important to make conscious decisions about a future path design.

In this paper we propose to follow a scientific research methodology to analyze the as-is situation of an enterprise in terms of interoperability requirements from an architectural point of view. But first of all, we need to know what interoperability is. The most commonly acknowledged definition of interoperability is the one provided by IEEE, considering interoperability as the ability of two or more systems or components to exchange information and to use the information that has been exchanged [5]. However, interoperability is not easy to understand due to its numerous definitions and interpretations. Ford et al. point out that according to their survey, thirty-four definitions of interoperability have been proposed since 1977 [6].

To deal with this problem the Ontology of Interoperability (OoI) was proposed to define the Interoperability domain [7. It was thereafter extended by the Ontology of Enterprise Interoperability (OoEI). This OoEI aims at formally defining Enterprise Interoperability (EI) while providing a framework to describe problems and related solutions pertaining to the interoperability domain. It was proposed in the general context of EI with high level concepts using the system theory [8]. This needs to be enriched with enterprise concepts from EA domain. Moreover, EA models do not take into account interoperability concepts despite the importance of the interoperability in the survival cycle of an enterprise. In this paper we propose to integrate the two domain concepts (EA and EI) from a business point of view and to use the conceptual modeling to define the integrated domain. It is widely accepted that conceptual models are a prerequisite for successfully planning and designing complex systems [9]. Over the last decades, conceptual modeling has been employed to facilitate, systematize, and aid the process of information system engineering [10].

Based on ArchiMate as a modeling language of EA [11] and OoEI 12, a conceptual model is proposed. A conceptual model is a typically graphical representation, hence can provide limited vocabulary [13, constructed by IS professionals of some-ones or some groups perception of a real-world domain 14. Conceptual modeling may be used to ease the implementation of an information system or to provide a common understanding between the organizations needs and an enterprise application. It is also suitable to systematize knowledge, provide guiding research and map a portion of reality [14].

The proposed model is defined based on the design-science research (DSR) methodology proposed by Hevner et al. [15] and Winter [16]. DSR addresses important unsolved problems in unique or innovative ways or solved problems in more effective or efficient ways comparing to traditional or so-called routine design [15]. Although it may seem impossible to find general and meaningful concepts for the entire domain of integrated Enterprise Architecture Interoperability, it is better to adopt the so-called constructive research strategy [17. 


\section{Research Methodology}

This research is based on a simplification of the design-science research (DSR) as proposed by Hevner et al. [15] and Winter [16. The methodology applied is divided according to the two processes of design science research in information system, Build and Evaluate [17]. The build process is composed by two stages whereas and the evaluation process is composed by only one stage (see Table 1).

Table 1. Research methodology

\begin{tabular}{|l|l|l|}
\hline \multicolumn{2}{|c|}{ Build } & Evaluate \\
\hline Conceptual definition & Conceptual model construction & Evaluation \\
\hline - Domain definition & - Analysis of relations between concepts & Use case \\
- Concepts identification & - Integration of the two domains concepts & \\
- Concept definition & into one model & \\
\hline
\end{tabular}

The first stage, conceptual definition, has two main milestones: concepts domain and domain definitions within the set up boundaries established between EI and EA. In this stage we proceed with literature study on interoperability models and frameworks together with EA modeling languages. Also, at this stage, we identify and define the concepts that we present in section 3. An analysis of the relations between concepts is required to understand the integrated model that is constructed in section 4 .

The second stage, evaluation, is done based on the observational case study as described in [15]. The evaluation part is illustrated by a case study in section 5 .

\section{Conceptual Definition}

\subsection{Enterprise Interoperability}

In order to understand the enterprise interoperability domain, we need to study the operational entities where interoperations take place within an enterprise. This aspect is mainly defined through various existing interoperability frameworks and models, which are reviewed as follows:

Framework for Enterprise Interoperability. The main purpose of an interoperability framework is to provide an organizing mechanism so that concepts, problems and knowledge on enterprise interoperability can be represented in a more structured way [18].

So far, the most known EI frameworks are: ATHENA (Advanced Technologies for interoperability Heterogeneous Enterprise Networks and Applications) Interoperability Framework (AIF) [19, the European Interoperability Framework 20] and the Framework for Enterprise Interoperability (FEI) [21. 
Compared to other interoperability frameworks, the FEI provides three explicitly defined interoperability dimensions (interoperability barriers, interoperability concerns and interoperability approaches) to allow defining interoperability research domain [12. The FEI was developed within the frame of INTEROP Network of Excellence [18, and is now published as an international standard (ISO 11354 - 1). It defines a classification scheme for interoperability knowledge according to three dimensions:

1. Interoperability Barriers: According to FEI, the establishment of interoperability consists in removing all the identified barriers. Three kinds of barriers are identified: Conceptual (syntactic and semantic differences of information to be ex-changed), Technological (incompatibility of information technologies: architecture and platforms, infrastructure, etc.), and Organizational (definition of responsibilities and authorities).

2. Interoperability Concerns: They represent the areas concerned by interoperability in an enterprise. Four concerns are defined, namely business interoperability (work in a harmonized way to share and develop business between companies despite the difference of methods, decision making, culture of enterprises, etc.), process interoperability (make various processes work together. In the inter-worked enterprise, the aim will be to connect internal processes of two companies to create a common process), service interoperability (making work together various services or applications by solving the syntactic and semantic differences) and data interoperability (make work together different data models with different query languages to share information coming from heterogeneous systems).

3. Interoperability Approaches: there are three basic ways to relate entities together to establish interoperations: The integrated approach (characterized by the existence of a common format for all the constituents systems), the unified approach, characterized by the existence of a common format but at a metalevel, the federated approach, in which no common format is defined. This approach maintains the identity of interoperating systems; nothing is imposed by one party or another and interoperability is managed in an ad-hoc manner.

The Ontology of Enterprise Interoperability (OoEI). The approach adopted for building the OoEI considers interoperability from a problem-solving perspective, not restricted to communication matters. Indeed, contrary to what can be found in most of the available definitions, interoperability is not only related to communication. The components of a system do not necessary have to communicate, but might simply have to be composed together for a specific purpose. This is illustrated by the following definition: An interoperability problem appears when two or more incompatible systems are put in relation. Interoperability per se is the paradigm where an interoperability problem occurs [7. Based on an analysis on the Enterprise frameworks and existing interoperability models [12, the OoEI was defined as depicted by the figure 1 .

Interoperability is implemented as a subclass of the Problem concept. Problems of interoperability exist when there is a relation, of any kind, between 


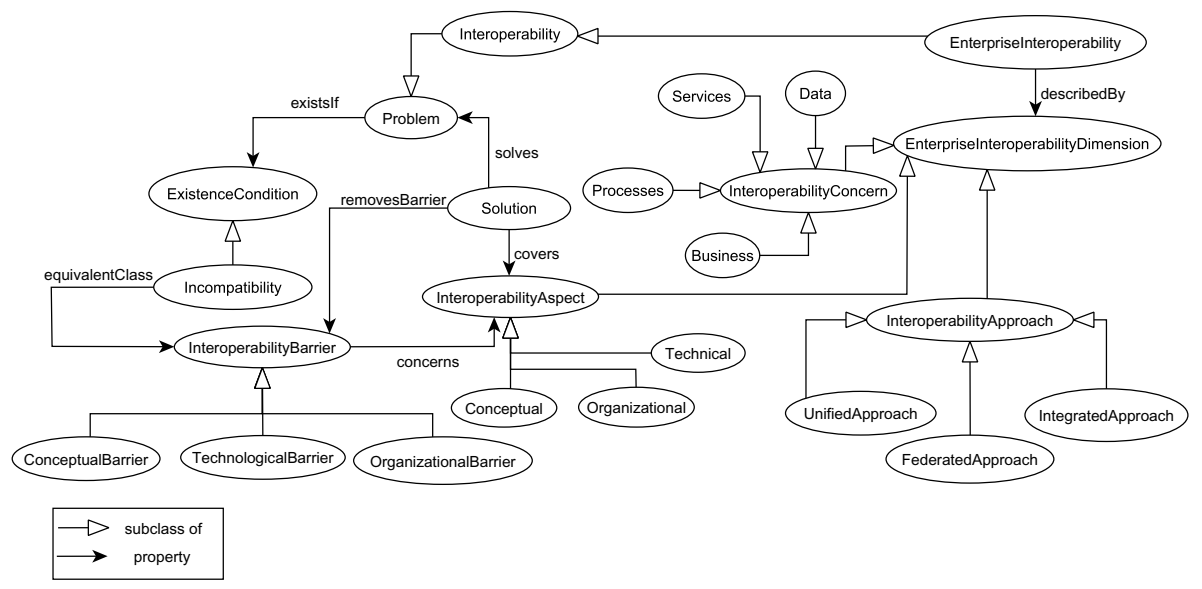

Fig. 1. Extract of OoEI

incompatible systems in a super- system they belong to or system they will form. Incompatibility concept is a subclass of a more generic InteroperabilityExistenceCondition class aiming at explicitly formalizing the fact that Incompatibility is the source of interoperability problems for systems of any nature, as soon as they belong to the same super-system and there is a relation of any kind between those systems.

Three main dimensions of EI are considered: Interoperability aspects (conceptual, organizational and technical), Interoperating entities, also known as EI concerns (i.e. business, process, service and data) and Interoperability approaches (integrated, unified and federated). These are represented by the three concepts: InteroperabilityAs-pect, InteroperabilityApproach, and InteroperabilityConcern respectively. These are all modeled with their different constituents represented here as instances under the EnterpriseInteroperabilityDimension concept, as shown in figure 1.

Interoperability problems are represented by the InteroperabilityBarrier concept. The term barrier is defined as an incompatibility, obstructing the sharing of information and preventing exchanging services 21. It is then assimilated (with the equivalentClass in figure 1) to the Incompatibility concept. The establishment of interoperability (with its three aspects) consists of removing identified barriers (conceptual barrier, organizational barrier or/and technological barrier). Hence each InteroperabilityBarrier is related to the corresponding InteroperabilityAspect.

Relevant Concepts in Enterprise Interoperability. Dealing with enterprise interoperability requires consideration of the enterprise from a general perspective, taking into account not only its different components and their interactions but also the environment in which it evolves and the interface through which it communicates with its environment. 
The interface is a system's element through which a connection between the system and its environment can be established. It also represents the system's boundaries. The interfaces are important for developing interoperability and avoiding interoperability problems. An enterprise is considered as a complex system in the sense that it has both a large number of parts and the parts are related in ways that make it difficult to understand how the enterprise operates and to predict its behavior The establishment or diagnosis of enterprise interoperability leads to identify the different operational levels that are concerned. Four enterprise levels are defined in the FEI, namely business, process, service and data. They represent the areas concerned by interoperability in the enterprise.

- Interoperability of data aims to make work together different data models with different query languages to share information coming from heterogeneous systems.

- Interoperability of services aims at making it possible for various services or applications (designed and implemented independently) to work together by solving the syntactic and semantic differences.

- Interoperability of processes aims to make various processes work together. In the interworked enterprise, the aim will be to connect internal processes of two companies to create a common process.

- Interoperability of business aims to work in a harmonized way to share and develop business between companies despite the difference of methods, decision making, culture of the enterprises or, the commercial making.

According to 21, there are three kinds of interoperability problems, called also barriers that enterprises may face: conceptual, technological or organizational.

- Conceptual problems are mainly concerned with the syntactic and semantic incompatibilities of information to be exchanged or to be used during an interoperation. These problems concern the modeling at the higher level of abstraction (i.e. enterprise models) as well as the level of programming (i.e. low capacity of semantic representation of XML). Syntactic differences can be found whenever different structures are used to represent information and knowledge.

- Technological problems refer to the use of computer or ICT (Information and Communication Technologies) to communicate and exchange information (i.e. architecture and platforms, infrastructure). These problems concern the standards to use, store, exchange, processes or computerize information.

- Organizational problems relate to the definition of responsibilities and authorities so that interoperability can take place under good conditions. Responsibility needs to be defined in order to delegate tasks (process, data, software, computer). If responsibility in an enterprise is not clearly and explicitly defined, interoperation between two systems is obstructed. Authority is an organizational concept which defines who is authorized to do what. For example, it is necessary to define who is authorized to create, modify, maintain data, processes, services, etc. 


\subsection{Enterprise Architecture}

Architecture is a consistent whole of principles, methods and models that are used in the design and realization of organizational structure, business processes, information systems, and infrastructure [22. The use of an enterprise architecture helps to chart the complexity of an organization. The specification and description of organizations components and especially their relationships in architecture requires a coherent architecture modeling language.

Overview of EA Modeling Languages. The objective here is not to provide an exhaustive review of existing EA modeling languages but rather to present relevant modeling languages that are selected specifically for the purpose of this work. So far, the most known EA modeling languages are the Unified Enterprise Modeling Language (UEML), Design and Engineering Methodology for Organizations (DEMO) and ArchiMate.

The UEML is an on-going attempt to develop theories, technologies and tools for integrated use of enterprise and IS models expressed using different languages. By this we mean keeping the existing models as they are and establish relationships between them in an explicit and usable way, supporting, e.g., consistency checking, automatic update reflection, model-to-model translation and other services across modeling language boundaries. UEML is thus intended as an intermediate language - or a hub - through which different languages can be connected, thereby facilitating a web of languages and of models expressed in those languages [23].

DEMO is a method comprising of a comprehensive set of conceptual modeling techniques, in combination with a theory based way of thinking and associated way of working, focused on modeling, analyzing and designing the essential aspects of an organization [24. DEMO uses the word essential here to refer to the implementation-independent aspects of an organization. As such, DEMO aims to abstract away from implementation-specific details, such as the information systems present in business collaboration [25].

ArchiMate is an Open Group standard [11] for the modeling of enterprise architectures , emphasizing a holistic view of the enterprise. This means that architects can use ArchiMate to model, amongst others, an organization's products and services, how these products and services are realized/delivered by business processes, and how in turn these processes are supported by information systems and their underlying IT infrastructure. ArchiMate is geared towards Information processing dominant organizations such as banks, insurance companies, government agencies, etc. [11.

Compared to other EA modeling languages, ArchiMate is successfully used and applied in many industrial cases [26]. Subsequently, we propose in this work to focus on ArchiMate and its main concepts. The choice of the language is motivated by the capacity of ArchiMate to distinguish between the structural or static aspect and the behavioral or dynamic aspect of enterprises. Moreover, our analysis will be limited to the ArchiMate business layer meta-model. As implied by name, the business layer focuses on an organization's business concepts such 
as products, (commercial) services, and business processes. The business architecture results from the implementation of business strategies and the definition of processes. The functional requirements of business process support systems, i.e. the information systems that will operationally support the business, are derived from this architecture [26].

Note that, the same approach could be applied with any other EA modeling language following the same research methodology.

ArchiMate and Its Main Concepts. In 22, three main layers are defined:

1. The Business layer offers products and services to external customers, which are realized in the organization by business processes performed by business actors.

2. The Application layer supports the business layer with application services which are realized by (software) applications.

3. The Technology layer offers infrastructural services (e.g., processing, storage and communication services) needed to run applications, realized by computer and communication hardware and system software.

Our analysis concerns the ArchiMate business layer meta-model. Figure 2 provides an excerpt of the ArchiMate business layer concepts and their relations. Note that we use only an excerpt of the ArchiMate business layer meta-model so as to focus on those concepts and relations relevant for interoperability. The ArchiMate business layer meta-model concepts, adapted from [11,22] are described as follows:

- Business actor: It defines an individual persons (e.g., customers or employees), but also groups of people (e.g., departments or business units) within the organizations.

- Business role: A role that an actor fulfills in an organization. Importantly, this role is usually defined as the work carried out by an actor.

- Business collaboration: It defines a (temporary) configuration of two or more business roles resulting in specific collective behavior in a particular context.

- Organizational service: It is a unit of functionality that is meaningful from the point of view of the environment. The following concepts realize a service: Busi-ness processes, business functions, business interactions. Moreover, A business process/function is a unit of internal behavior, performed by one or more roles within the organization. Finally A business interaction is a unit of behavior similar to a business process or function, but it is performed in a collaboration of two or more roles within the organization.

- Business event: An event that happens (externally) and may influence business processes, functions or interactions. A business event is most commonly used to model something that triggers behavior, but other types of events are also conceivable: e.g., an event that interrupts a process.

- Business object: An entity manipulated by behavior such as business processes or functions. 


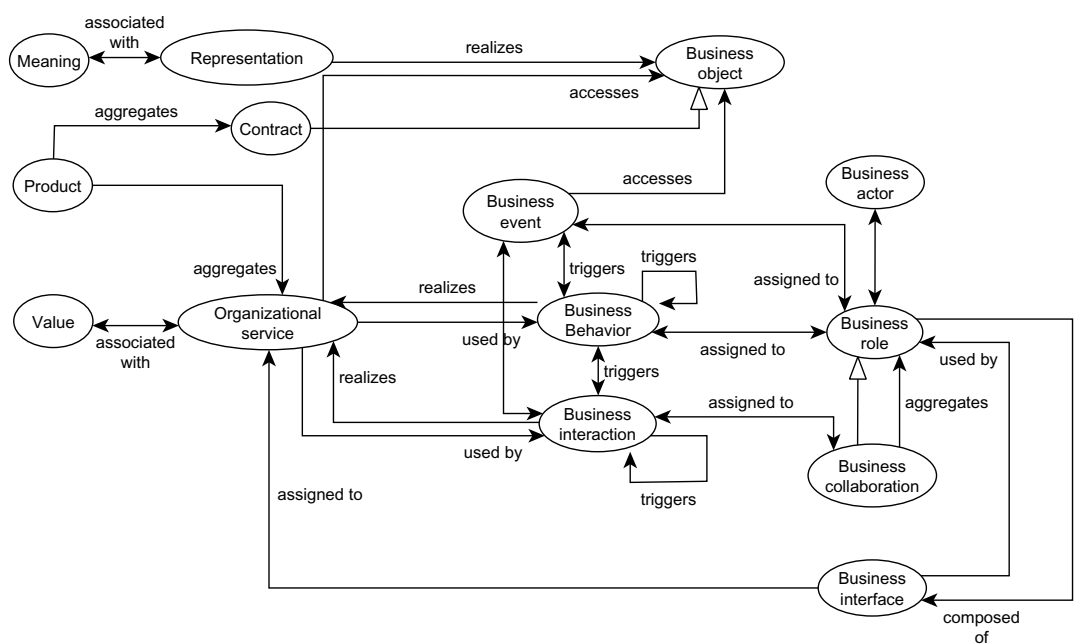

Fig. 2. ArchiMate business layer concepts

\section{Conceptual Model Construction}

\subsection{Enterprise Architecture and Interoperability}

ArchiMate, as presented in section 3.2, does not consider properly interoperability concepts. In the meanwhile, we may found concepts and relations exploring potential collaboration/interactions with local or external units to the main organization that may be a source of interoperability [5,22. Subsequently, this emphasizes the motivation of bridging the gap between both research areas EA and EI. Moreover, it presents tendency research topics for networked enterprises dealing with current challenges such as economic changes, globalization, et cetera. On one hand, interoperability framework needs EA models to analyze current situation and path forward future design for enterprises. On the other hand, EA models will be enriched with interoperability concepts to tackle new networked enterprises requirements (e.g., outsourcing).

Being "interoperable" refers to being able to share information between business partners, understand and process exchanged data, seamlessly integrate it into internal ICT systems, and enable its beneficial use [27]. In the following we propose the construction of the integrated model at the business layer. As implies by name, the business layer focuses on business concepts such as services, business process, product, etc. From the interoperability side, we focus also on business interoperability that can be defined as the organizational and operational ability of an enterprise to cooperate with its business partners and to efficiently establish, conduct and develop IT-supported business relationships with the objective to create values [27]. 


\subsection{Integration of ArchiMate EA Concepts into OoEI}

In this section, we define the integrated enterprise architecture interoperability model at the business layer. Figure 3 gives an overview of this model.

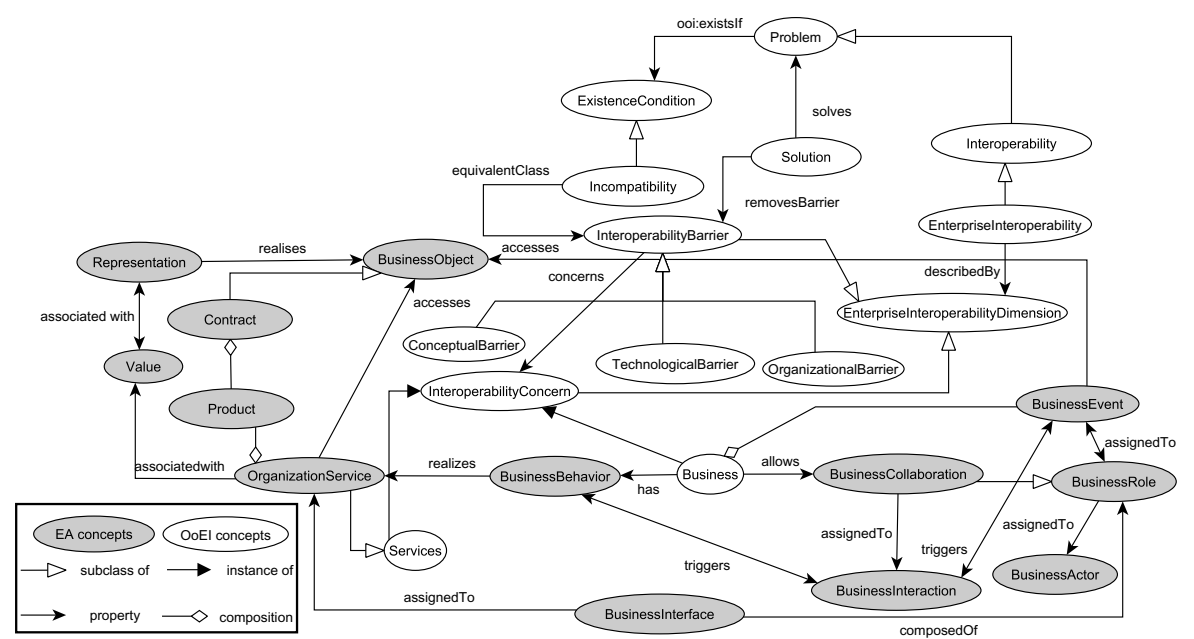

Fig. 3. Integrated Model: EA concepts and interoperability at business layer

In order to differentiate the EA concepts from the interoperability ones, we present them with the gray color.

Business collaboration, that defines a configuration of two or more business roles, will not be possible if the two roles are incompatible. Hence the importance of the interoperability that allows this kind of collaboration. This is designed by the property allows between the concepts business and businessCollaboration.

The two main dimensions of EI: interoperability barriers and interoperability concerns are considered. Hence we find the concepts InteroperabilityBarrier and InteroperabilityConcern as well as their respective sub-classes: OrganizationalBarrier, TechnologicalBarrier, ConceptualBarrier and Business, Process, Service, and Data.

The business interoperability concern has a behavior allowing it to realize a service. Hence the concept Business is related to BusinessBehavior by "has" property and the BusinessBehavior is related to OrganizationService by "realize" property.

\section{Evaluation Using a Case Study}

As part of the research approach, this section illustrates the evaluation of the proposed model using an industrial case study. The use case concerns a multinational company: METS (Manufacture Electro-Technical of Sousse) a subsidiary of the 
German Draxelmaer group 1 , specialized in automobile manufacturers including wiring harness systems, exclusive interiors and electrical components. To ensure its functions and reach its objectives, the company needs to interoperate with many partners, including its headquarters in Germany. This is relevant for the application of our approach.

In order to understand the way the enterprise functions, a series of interviews were conducted, our integrated model was then instantiated using the information of the company. METS is a $100 \%$ export oriented company: all its production is directed to the headquarters in Germany. The headquarters are then responsible for the distribution to the clients or other production sites. The normal business process starts when the company receives an order of production from the headquarters in Germany. If the order concerns a new product, then a prototyping is needed and a sample is produced. After a decision is reached, the production process can be launched. There are five main stakeholders for the company:

- The headquarters in Germany, from where the company receives orders.

- The production site in Poland to whom the company exports the semi-final prod-ucts.

- The production sites, from where the company receives semi-final products to finalize.

- The suppliers of the raw materials and accessories.

- Customs for the export.

As analyzing relations are the first requirement for identifying interoperations, a formal representation of the METS Company and the main relations are provided, using the integrated model. In order to differentiate between the instantiated concepts (i.e. specific to the company) and those of the proposed model, the instantiated concepts are represented by rectangle shapes as shown in figures 4 and 5 .

The company is represented by the Enterprise_Mets concept. As an instance of ooei:System, it inherits all their properties and constituents. Hence it has its own structure and behavior, represented respectively by Structure_METS and Behavior_METS. The company produces wire harnesses for the cars and has two main objectives: continuous reduction of the costs of its production and to be the leader within its market. This is represented by the concept Harness_production, instance of ooei:function and two instances ooei:objective: Market_leader and Reduce_costs.

As any multinational enterprise, METS evolves in its environment and has many partners. This is represented by Mets_env concept, instance of ooei:environment. Within this environment, the customs, the supplier of the accessories, the transporter, the Draxelmaier headquarters, the supplier of raw material and the provider of all other services are found. This is respectively represented by the concepts: Customs, Ac_supplier, Transporter, Drx_group,

${ }^{1}$ http://www.draexlmaier.de 

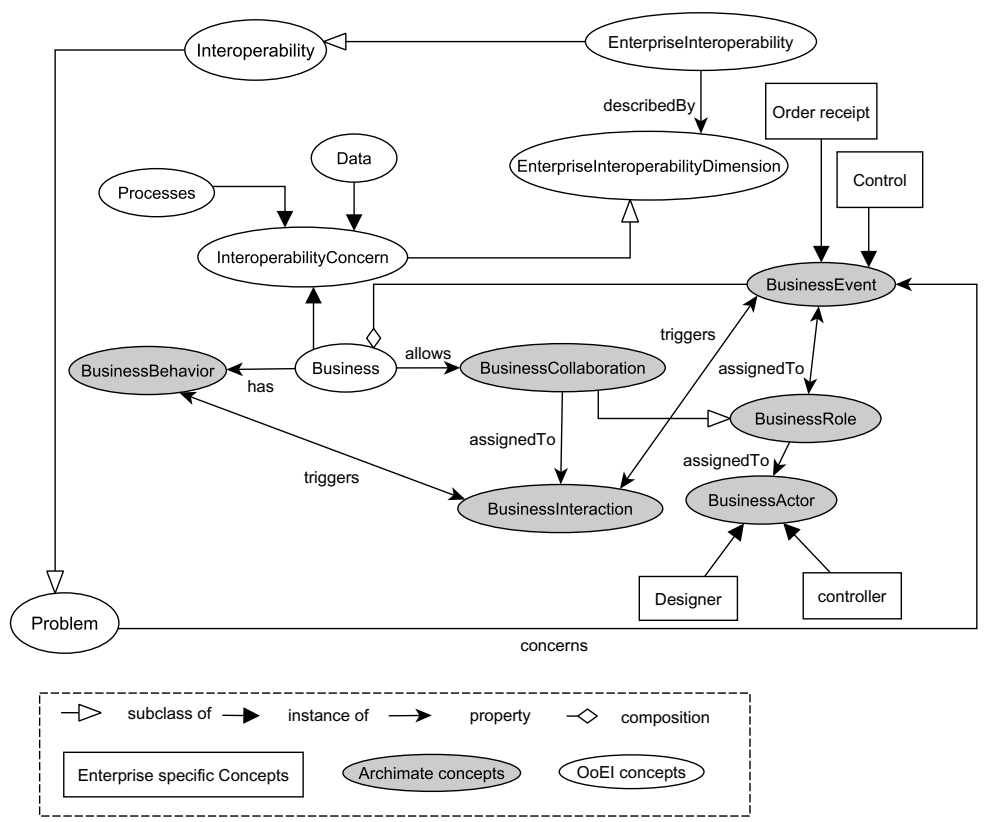

Fig. 4. Use case application - Business layer modeling

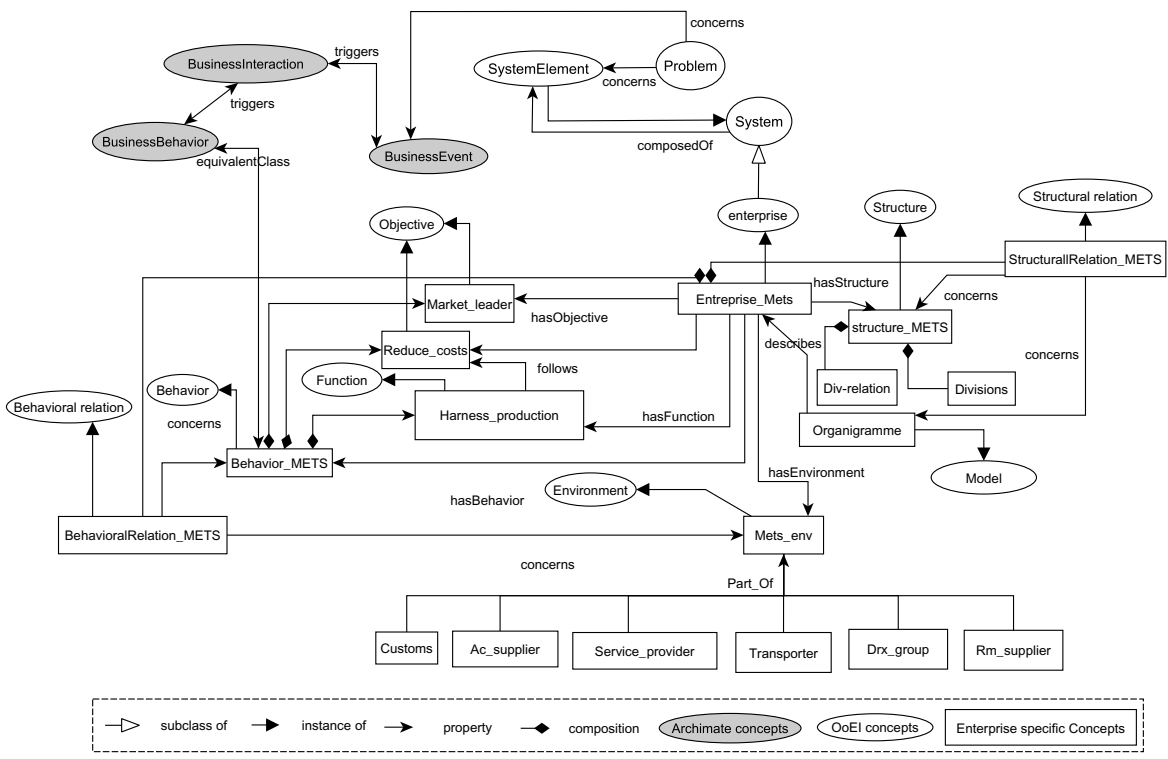

Fig. 5. Use case application- Company specification 
Rm_supplier, Service_provider. The instantiation of the integrated model provides an overview of the enterprise structure and the main relations that exist.

The case study evaluation provides primer results. The maturity of this evaluation is, certainly, limited but offers an initial investigations for interoprabilty readiness in networked enterprises. Back to our scenario, we observed that based on the resulted model, the managers can have a clear idea about the main elements within their architecture when dealing with interoperability requirments. This can be thereafter extended by associated rules to allow automatic reasoning for developing tools for diagnosis of enterprise interoperability problems.

\section{Conclusion and Future Work}

In this paper, we have proposed an integrated model bridging the interoperability and enterprise architecture concepts following the constructive research methodology. At a first stage, the concepts were identified and defined, and then the analysis of the relations between concepts was defined in order to construct the integrated conceptual model. The evaluation stage was done through a case study. The resulted integrated model was based on the OoEI and the Archimate model at the business layer.

Future work are planned to extend the defined conceptual model with concepts from other EA modeling languages and to cover applicative and technical levels [4] of the enterprise architecture.

\section{References}

1. Dinh, T.L., Thi, T.T.P.: Information-driven framework for collaborative business service modelling. IJSSMET 3(1), 1-18 (2012)

2. Architectures for enterprise integration and interoperability: Past, present and future. Computers in Industry 59(7), 647-659 (2008)

3. Vernadat, F.: Enterprise Integration Modeling and Integration: Principles and Applications. Springer (1996)

4. Lankhorst, M.M.: Enterprise Architecture at Work - Modelling, Communication and Analysis, 4th edn. The Enterprise Engineering Series. Springer (2013) 978-3642-29650-5

5. Institute of Electrical and Electronics Engineers. IEEE standard computer dictionary: A compilation of ieee standard computer glossaries (1990)

6. Ford, T., et al.: Measuring System Interoperability: An i-Score Improvement. In: Proceedings of the 6th Annual Conference on Systems Engineering Research (2008)

7. Naudet, Y., Latour, T., Chen, D.: A systemic approach to interoperability formalization. In: IFAC 2008 Workshops (2008)

8. Von Bertalanffy, L.: General System Theory: Foundations, Development, Applications. Georges Braziller, Inc., New York (1968)

9. Moody, D.L., Shanks, G.G.: Improving the quality of data models: empirical validation of a quality management framework. Inf. Syst. 28(6), 619-650 (2003)

10. Recker, J.: Conceptual model evaluation. towards more paradigmatic rigor. In: Proceedings CAiSE 2005 Workshops, Porto, Portugal (2005) 
11. Jonkers, H., Proper, H.A., Turner, M.: TOGAF and ArchiMate: A Future Together. White Paper W192. The Open Group (November 2009)

12. Guédria, W.: A Contribution to Enterprise Interoperability Maturity Assessment. Ph.D. thesis, University of Bordeaux1, Bordeaux, France (2012)

13. Schermann, M., Böhmann, T., Krcmar, H.: Explicating design theories with conceptual models: Towards a theoretical role of reference models. In: issenschaftstheorie und gestaltungsorientierte Wirtschaftsinformatik, pp. 175-194 (2009)

14. Shanks, G., Tansley, E., Weber, R.: Using ontology to validate conceptual models. Commun. ACM 46(10), 85-89 (2003)

15. Hevner, A.R., March, S.T., Park, J., Ram, S.: Design science in information systems research. MIS Q. 28(1), 75-105 (2004)

16. Winter, R.: Design science research in europe. European Journal of Information Systems - Eur. J. Infor. Syst. 17(5), 470-475 (2008)

17. Vicente, P., Mira da Silva, M.: A conceptual model for integrated governance, risk and compliance. In: Mouratidis, H., Rolland, C. (eds.) CAiSE 2011. LNCS, vol. 6741, pp. 199-213. Springer, Heidelberg (2011)

18. Chen, D., Dassisti, M., Elvester, B.: Interop noe deliverable di.3: Enterprise interoperability framework and knowledge corpus - final report. Technical report, Interoperability Research for Networked Enterprises Applications and Software (INTEROP Network of Excellence), IST - Contract no.: IST-508 011 (2007)

19. AIF official website. ATHENA interoperability Framework (2004), http://www.modelbased.net/aif/wholesite.html

20. Miller, F.P., Vandome, A.F., McBrewster, J.: European Interoperability Framework. VDM Publishing (2011)

21. Chen, D.: Enterprise Interoperability Framework. In: Workshop on Enterprise Modelling and Ontologies for Interoperability (EMOI-INTEROP) (January 2006)

22. Jonkers, H., Lankhorst, M., Van Buuren, R., Bonsangue, M., Van Der Torre, L.: Concepts for modeling enterprise architectures. International Journal of Cooperative Information Systems 13, 257-287 (2004)

23. Vernadat, F.: Ueml: towards a unified enterprise modelling language. International Journal of Production Research 40(17), 4309-4321 (2002)

24. Dietz, J.L.G.: Enterprise ontology: theory and methodology. Springer (2006)

25. Kinderen, S., Gaaloul, K., Henderik, A.: Bridging value modelling to ArchiMate via transaction modelling, pp. 1-15 (2012)

26. Sanchez, A., Basanya, R., Ojo, A.K., Janowski, T.: Enterprise architectures - enabling interoperability between organizations. In: 8th Argentinean Symposium on Software Engineering, part of 36th Argentine Conference on Informatics, ASSE 2007, Mar del Plata, Argentina (August 2007)

27. Legner, C., Wende, K.: Towards an excellence framework for business interoperability (2006) 